\title{
RANKING DE FORNECEDORES EM UMA CADEIA DE SUPRIMENTOS DE MATERIAL HOSPITALAR DA MARINHA DO BRASIL UTILIZANDO PARÂMETROS DO MODELO SCOR 12.0: UMA ABORDAGEM MULTICRITÉRIO
}

\author{
Luiz Frederico Horácio de Souza de Barros Teixeira \\ Centro de Análises de Sistemas Navais (CASNAV)/ Universidade Federal Fluminense (UFF) \\ Praça Barão de Ladário, s/nº Ilha das Cobras, Rua da Ponte, Ed. 23, Centro, Rio de Janeiro \\ - RJ / Rua Passo da Pátria, 156 - 209, São Domingos, Niterói - RJ \\ frederico.horacio@gmail.com \\ Priscilla Cristina Cabral Ribeiro \\ Universidade Federal Fluminense (UFF) \\ Rua Passo da Pátria, 156 - 209, São Domingos, Niterói - RJ \\ priscillaribeiro@id.uff.br \\ Carlos Francisco Simões Gomes \\ Universidade Federal Fluminense (UFF) \\ Rua Passo da Pátria, 156 - 209, São Domingos, Niterói - RJ \\ cfsg1@bol.com.br

\section{Marcos dos Santos} \\ Centro de Análises de Sistemas Navais (CASNAV) / Instituto Militar de Engenharia (IME) \\ Praça Barão de Ladário, s/nº Ilha das Cobras, Rua da Ponte, Ed. 23, Centro, Rio de Janeiro \\ - RJ / Praça Gen. Tibúrcio, nº 80, Praia Vermelha, Urca, Rio de Janeiro - RJ \\ marcosdossantos doutorado uff@yahoo.com.br
}

\begin{abstract}
RESUMO
O estudo do gerenciamento da cadeia de suprimentos pode resultar em benefícios para todas as partes envolvidas. Uma ferramenta comumente utilizada nas análises das cadeias de suprimento é o Supply Chain Operations Reference (SCOR), que é um modelo de diagnóstico de referência para todas as etapas de uma cadeia. Uma cadeia de suprimentos de material hospitalar, por sua vez, possui particularidades que a diferenciam de outras áreas da indústria e do comércio. Este trabalho propõe uma avaliação de fornecedores de material hospitalar de uma unidade da Marinha do Brasil por meio do método ordinal multicritério de apoio à decisão SAPEVO-M. O método é bastante recente e pouco explorado na literatura desta linha de pesquisa. Uma pesquisa na base SCOPUS demonstrou um relativo ineditismo na correlação da cadeia de suprimento com métodos ordinais, como o SAPEVO-M utilizado neste artigo. A avaliação de fornecedores foi realizada por dois especialistas, por meio de plataforma online de aplicação do método SAPEVO-M, denominada SapevoWeb. Os resultados alcançados permitem uma análise da qualidade do serviço prestado por aquelas empresas, na medida em que não há grandes diferenças entre uma alternativa e a seguinte. Contudo, esta diferença se evidencia à medida que as mesmas se distanciam no ranking.
\end{abstract}

Palavra-chave: Cadeia de Suprimento; SCOR; Métodos Multicritério de Apoio à Decisão; Método Ordinal SAPEVO-M; Marinha do Brasil. 


\begin{abstract}
The study of supply chain management can yield benefits for all parties involved. A commonly used tool in supply chain analysis is the Supply Chain Operations Reference (SCOR), which is a reference diagnostic model for all steps in a chain. A hospital supply chain, in turn, has particularities that set it apart from other areas of industry and commerce. This paper proposes an evaluation of health material suppliers of a Brazilian Navy unit through ordinal multi-criteria decision aid method SAPEVO-M. This method is quite recent and little explored in the literature of this research line. A search in the SCOPUS database has shown a relative novelty in supply chain correlation with ordinal methods such as SAPEVO-M used in this paper. Supplier evaluation was carried out by two experts through the SAPEVO-M online application platform called SapevoWeb. The results achieved allow an analysis of the quality of service provided by these companies, as there are no major differences between one alternative and the next. However, this difference is evident as they move apart in the ranking.
\end{abstract}

Keywords: Supply Chain; SCOR; multi-criteria decision analysis; ordinal method SAPEVO-M; Brazilian navy.

\title{
Como Citar:
}

TEIXEIRA, Luiz Frederico Horácio de Souza de Barros; RIBEIRO, Priscilla Cristina Cabral; GOMES, Carlos Francisco Simões; SANTOS, Marcos dos. Ranking de fornecedores em uma cadeia de suprimentos de material hospitalar da Marinha do Brasil utilizando parâmetros do modelo SCOR 12.0: uma abordagem multicritério. In: SIMPÓSIO DE PESQUISA OPERACIONAL E LOGÍSTICA DA MARINHA, 19., 2019, Rio de Janeiro, RJ. Anais [...]. Rio de Janeiro: Centro de Análises de Sistemas Navais, 2019.

\section{INTRODUÇÃO}

Quando um paciente é atendido em uma clínica ou hospital, ele desconhece as necessidades para a realização daquele procedimento, por mais simples que ele seja. Entretanto, o processo de atendimento do paciente em um hospital é apoiado por uma série de atividades operacionais, incluindo gerenciamento e distribuição de material hospitalar para os locais de atendimento (MOONS; WAEYENBERGH; PINTELON, 2019).

Embora haja problemas enfrentados pela saúde pública, as Forças Armadas conseguem manter um serviço de qualidade para atender o seu pessoal. Na Marinha do Brasil (MB) este esforço ocorre por meio do Sistema de Saúde da Marinha (SSM). Segundo as Normas para Assistência Médico-Hospitalar da Marinha (BRASIL, 2012), o SSM é o conjunto organizado de recursos humanos, materiais, financeiros, tecnológicos e de informações, destinado a prover as atividades de saúde na MB. Este sistema atende os militares da Marinha da ativa, reserva, e seus respectivos dependentes. Dados públicos do Anuário Estatístico da Marinha do Brasil (ANEMAR) (BRASIL, 2018) - apontam que a MB em 2018 possuía mais de 210.000 servidores, entre civis e militares, ativos e inativos. Assim, para prover material hospitalar para este público é necessária uma gestão eficiente da cadeia de suprimentos.

Apesar de haver na literatura estudos diversos atestando que as práticas de gerenciamento da cadeia de suprimentos (GCS) podem resultar em redução de custos e vantagem competitiva, as organizações de saúde têm sido demasiadamente lentas para adotar esses procedimentos (ARONSSON; ABRAHAMSSON; SPENS, 2011). 
O Supply Chain Operations Reference (SCOR) é um modelo amplamente empregado para avaliação de desempenho das Cadeias de Suprimento, embora tenha natureza genérica. Para Sellitto et al. (2015), quando abordado no contexto do apoio à decisão, o modelo SCOR é comumente associado ao método multicritério AHP.

Neste artigo, propõe-se a utilização de um método ordinal multidecisor de apoio multicritério à tomada de decisão (SAPEVO-M) para responder à seguinte questão de pesquisa: como se pode avaliar fornecedores de material hospitalar de uma unidade da Marinha do Brasil? A empresa foco será o Centro de Obtenção da Marinha no Rio de Janeiro (COMRJ), que consiste no maior órgão de obtenção de material para a Marinha do Brasil, com distribuição em âmbito nacional (BRASIL, 2019).

No presente trabalho serão consideradas as métricas de desempenho do modelo SCOR como critérios de avaliação dos fornecedores da cadeia de suprimentos. A seleção dos fornecedores e sua avaliação foi realizada por dois especialistas, por meio da plataforma online SapevoWeb (www.sapevoweb.com), específica para aplicação do método SAPEVOM. A avaliação consistirá em comparações pareadas entre os diversos critérios, para a geração de pesos, e entre as alternativas em cada critério, para a ordenação dos fornecedores. O trabalho está dividido em cinco seções. Após a introdução, na seção 2 é realizada uma revisão da literatura sobre GCS e modelo SCOR e é apresentado, brevemente, o método SAPEVO-M. A seção 3 apresenta o método de pesquisa empregado. Em seguida a seção 4 detalha o caso particular estudado para exemplificar a proposta dos autores. E, finalmente, a última seção apresenta as conclusões deste trabalho.

\section{REVISÃO DA LITERATURA}

\subsection{Modelo SCOR (SUPPLY CHAIN OPERATIONS REFERENCE)}

O conceito de Cadeia de Suprimentos transcende a definição de logística, que, por sua vez, pode ser constatada como inserida em cada componente da cadeia em questão. Segundo o Council of Supply Chain Management Professionals (2019), “a logística seria a parte da cadeia de suprimentos que, basicamente, planeja, implementa e controla, de forma eficiente e eficaz o fluxo, armazenagem e informações de bens”. Se anteriormente a logística era vista como uma atividade de apoio, muitas vezes associada apenas a transporte e armazenagem, segundo Gomes e Ribeiro (2014) a logística agrega valor às funções de serviço ao cliente, localização, administração, transporte, armazenagem e informação à cadeia produtiva.

A Gestão da Cadeia de Suprimentos (GCS), por sua vez, seria uma evolução da gestão da logística, incluindo um valor estratégico, por permitir que as empresas alcancem resultados melhores do que viriam a obter se agissem de forma individualizada. Tal esforço de coordenação permite uma coadministração de recursos, redução de inventários e desperdícios e maximização de lucros (COOPER; LAMBERT; PAGH; 1997).

Na GCS as empresas buscariam a redução de custos operacionais, terceirizando algumas atividades e focando em suas atividades fim, nas quais podem obter um diferencial (GOMES; RIBEIRO, 2014).

O modelo SCOR foi criado por uma empresa de consultoria em 1996 e logo endossado pelo Supply Chain Council (SCC) como um padrão entre as gestões da cadeia de suprimento. Esta ferramenta permite aos usuários entenderem os processos envolvidos em uma organização de negócios e identificar as características vitais que levam à satisfação do cliente. Em 2014 houve a fusão entre o SCC e o a APICS (American Production and Inventory Control Society), criando-se a APICS SCC, organização sem fins lucrativos responsável por continuamente atualizar o modelo SCOR (APICS SCC, 2017).-

O modelo SCOR une conceitos de reengenharia de processos, benchmarking e 
aplicação das melhores práticas e permite à organização detectar oportunidades de melhorar a sua competitividade. Este modelo vincula processos de negócios, métricas de desempenho, práticas e habilidades pessoais a uma estrutura unificada (VELYCHKO, 2015; PEÑAOROZCO; RIVERA, 2017). O objetivo da adoção de padrões, como as fornecidas pelo modelo SCOR, é possibilitar que as empresas desenvolvam e mantenham processos em suas cadeias de suprimento (CS) que atendam a determinadas métricas de desempenho, integrando medidas de garantia de qualidade no processo (LI; SU; CHEN, 2011). O diagnóstico apresentado pelo modelo SCOR não se restringe apenas na avaliação de desempenho financeiro das cadeias de suprimentos, mas é, também, um apoio à decisão a outros aspectos de sustentabilidade ao longo da CS (NTABE et al., 2015).

Segundo a APICS SCC (2017), os atributos do modelo SCOR 12.0, são:

- Confiabilidade: capacidade de executar tarefas conforme o esperado, o que inclui a previsibilidade do resultado de um processo;

- Responsividade: velocidade na qual as tarefas são executadas e/ou produtos são oferecidos aos clientes;

- Agilidade: capacidade de reagir às influências externas e responder a mudanças no mercado para obter ou manter vantagem competitiva;

- Custos: custo de operar os processos da cadeia de suprimentos, incluindo trabalho, material, gestão e transporte, entre outros;

- Gestão Eficiente de ativos: capacidade de utilizar eficientemente ativos, incluindo a redução de estoque, otimização da capacidade e terceirização.

Uma pesquisa na base de dados SCOPUS, realizada em 25 de agosto de 2019, com as expressões "Supply Chain" e "Supply Chain Operations Reference" obteve, para o período de 2009-2019, um total de 602 documentos, dos quais 304 artigos. A distribuição anual destes artigos pode ser observada na Figura 1.

Figura 1 - Publicação de artigos por ano no período 2009-2019

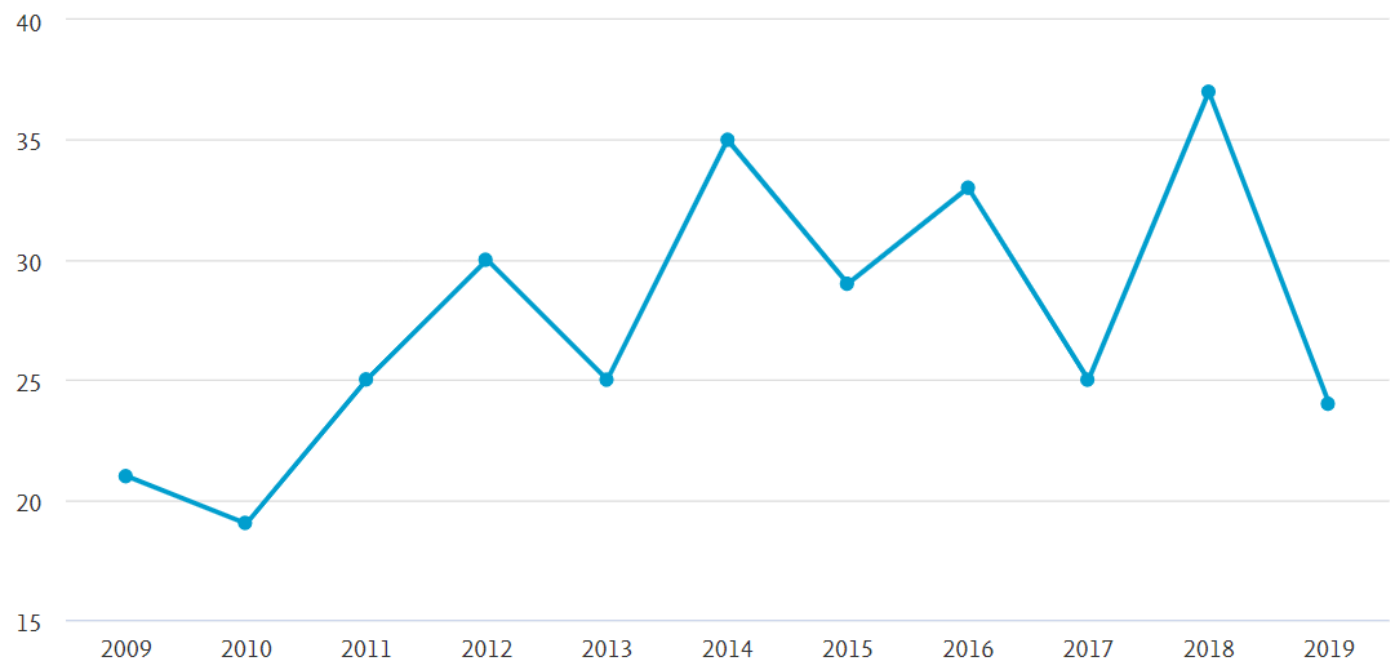

Documentos / Ano

Fonte: Elaboração própria (2019)

Este gráfico possui um desenho característico “dente de serra”, com uma sazonalidade cuja explicação pode estar associada ao lançamento de novos modelos. Por exemplo, houve uma redução no número de publicações em 2009 e um aumento a partir do 
final de 2010, que coincide com o lançamento do modelo SCOR 10.0 em dezembro daquele ano. Observa-se outra queda brusca no início de 2012 e um aumento significativo em 2013; provavelmente porque a versão 11.0 foi lançada no final de 2012. Em 2016 observa-se uma queda, crescendo novamente o número de publicações a partir de 2017, ano de lançamento da versão SCOR 12.0. Em 2019 verifica-se outra pequena queda que pode, porém, estar associada ao fato de os dados estarem incompletos por terem sido compilados até setembro do corrente ano. A Tabela 1 ilustra as datas de lançamentos dos modelos SCOR mais recentes.

Tabela 1 - Data de lançamento dos modelos SCOR

\begin{tabular}{|l|c|}
\hline Versão & Lançamento \\
\hline SCOR 8.0 & Junho / 2006 \\
\hline SCOR 9.0 & Novembro / 2008 \\
\hline SCOR 10.0 & Dezembro / 2010 \\
\hline SCOR 11.0 & Dezembro / 2012 \\
\hline SCOR 12.0 & Outubro / 2017 \\
\hline
\end{tabular}

Como pode ser observado na Figura 2, a China lidera as publicações neste período, com 69 artigos, seguida pelos Estados Unidos, com 37 e Reino Unido, com 21. A predominância chinesa também pode ser constatada entre as instituições que mais publicaram, conforme ilustrado pela Figura 3. O Brasil aparece na $4^{a}$ posição em número de publicações, contabilizando 20 artigos no período, com destaque para a Universidade de São Paulo (USP), que concentrou 5 destes.

Figura 2- Distribuição dos artigos por países

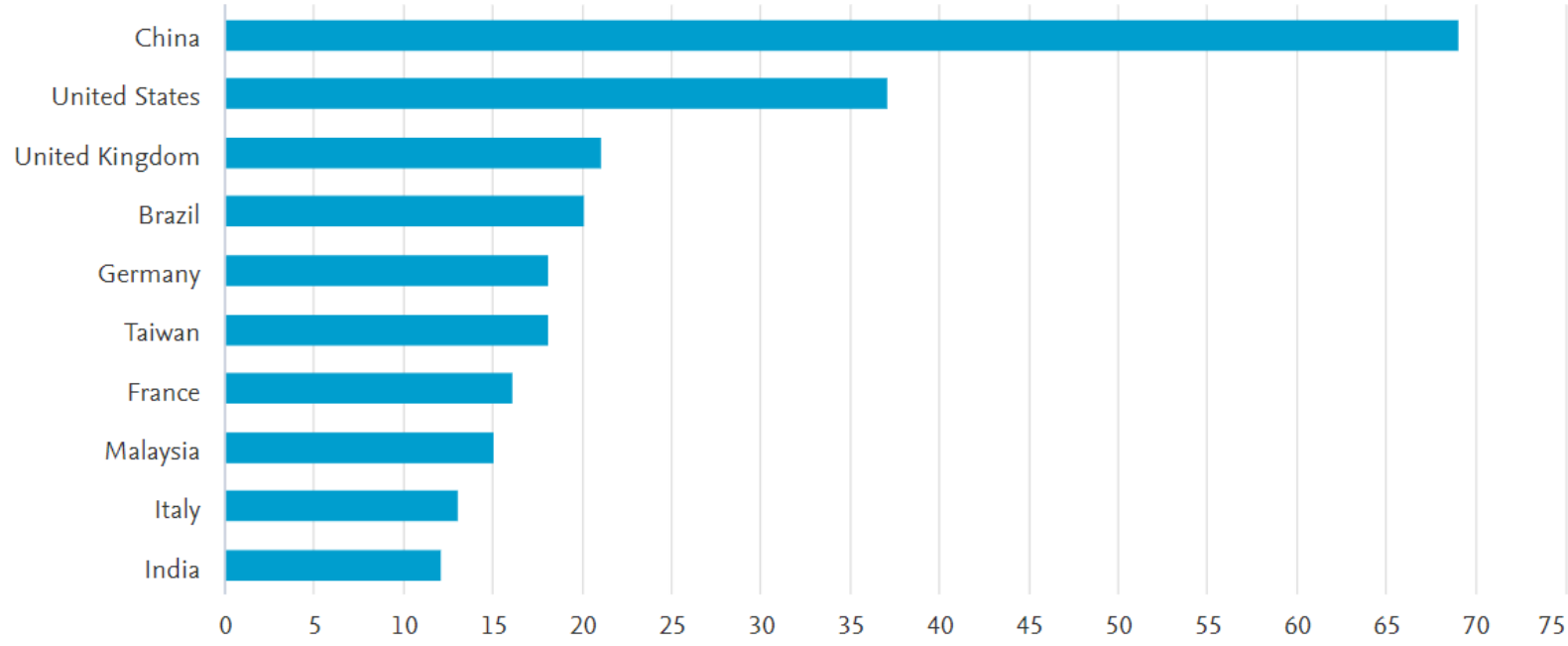

País / Documentos

Fonte: Elaboração própria (2019) 
Figura 3- Distribuição dos artigos por instituições

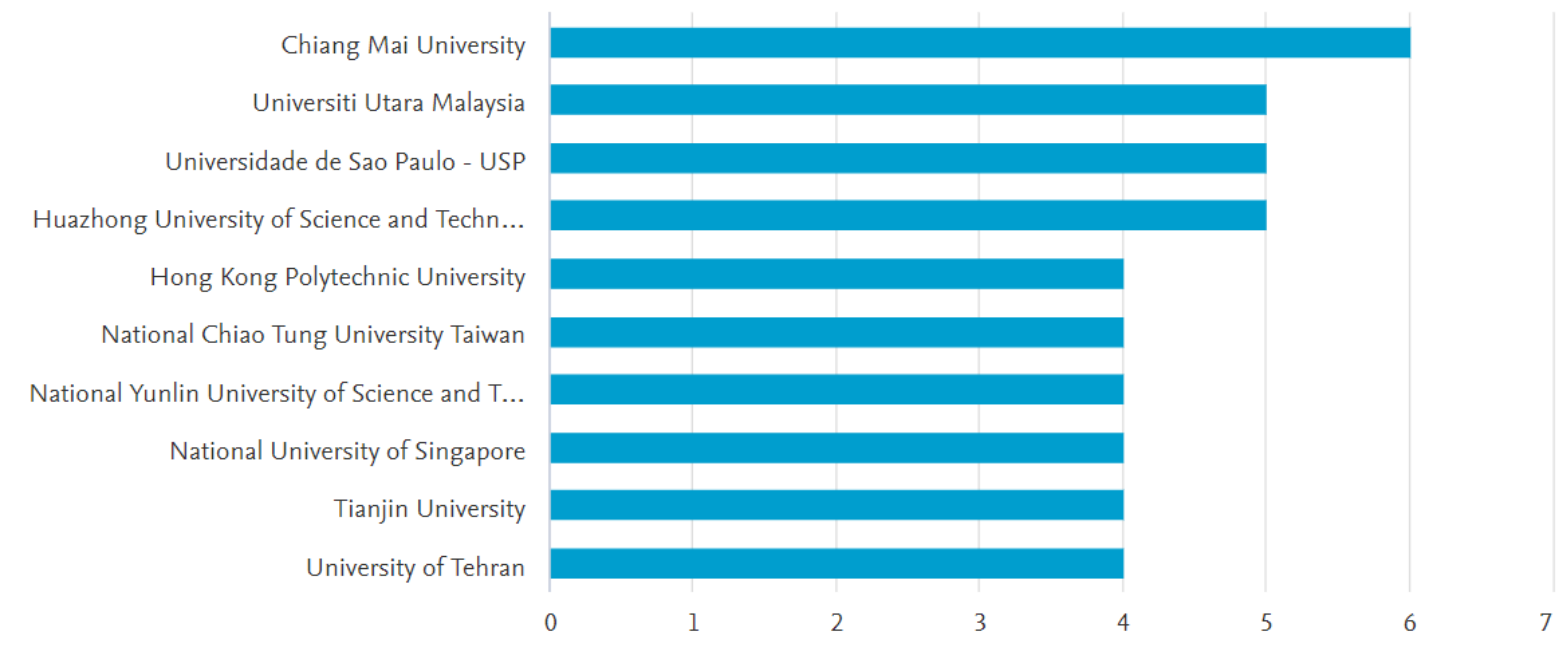

Instituição / Documentos

Fonte: Elaboração própria (2019)

Os autores que mais publicaram, na linha de pesquisa, estão destacados na Figura 4. Verifica-se que não há autor algum que tenha se destacado no período. O autor que mais publicou participou de quatro artigos sobre o tema no intervalo amostral de 10 anos, seguido por outros seis autores com três publicações cada.

Figura 4- Distribuição dos artigos por autores

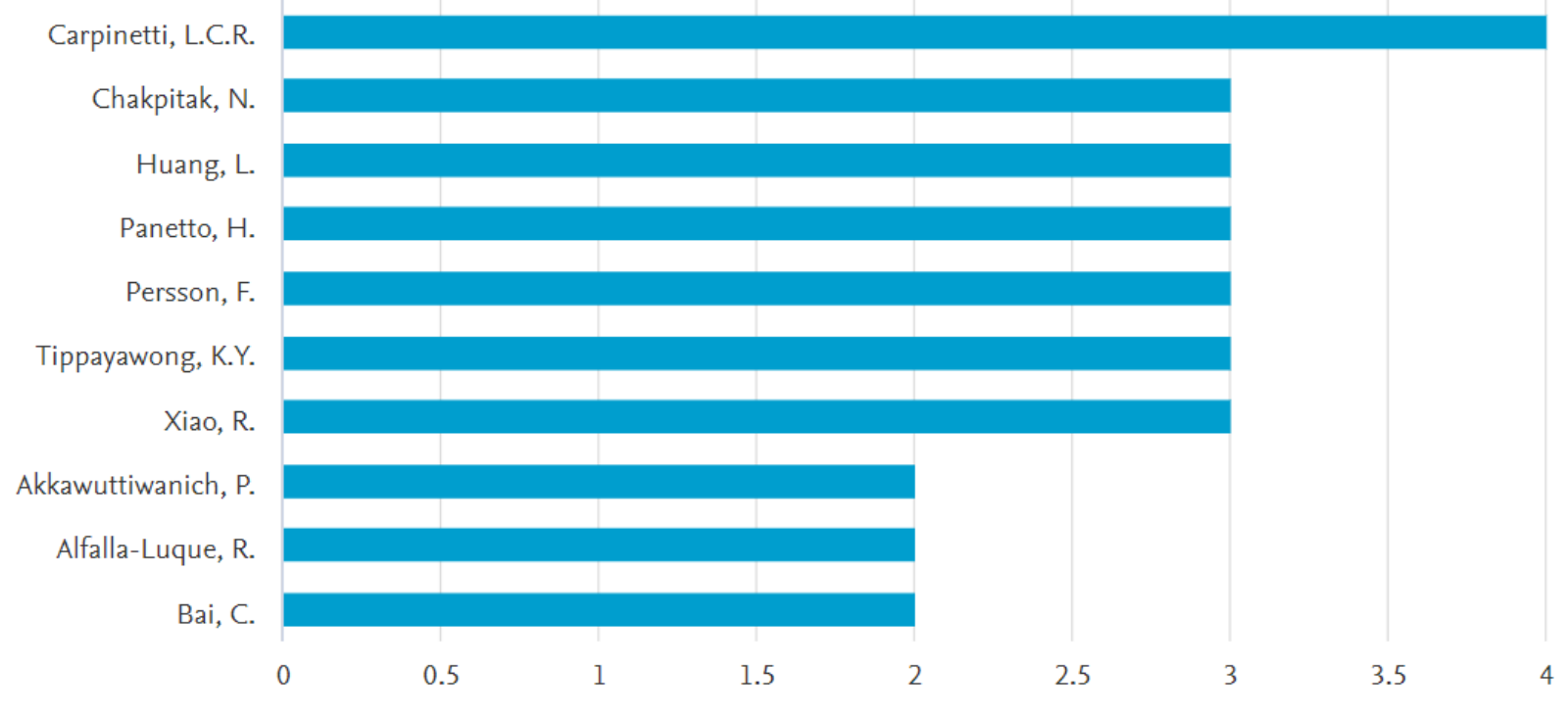

Autor / Documentos

Fonte: Elaboração própria (2019)

A Figura 5 complementa essa análise com o perfil dos periódicos que tiveram mais artigos publicados no período. Observa-se, também, uma distribuição homogênea entre os periódicos. Cabe ressaltar que alguns periódicos apresentaram um comportamento "dente de 
serra” semelhante à distribuição anual total de artigos sobre o tema, ilustrada na Figura 1. Verifica-se, inclusive, uma coincidência entre os períodos “de pico” em ambos os gráficos, ou seja, após o lançamento de um novo modelo SCOR ocorre, provavelmente, um boom de publicações, então os periódicos são mais suscetíveis àquela novidade e o número total de artigos no ano cresce.

Figura 5- Distribuição dos artigos por periódicos

\section{Documentos}

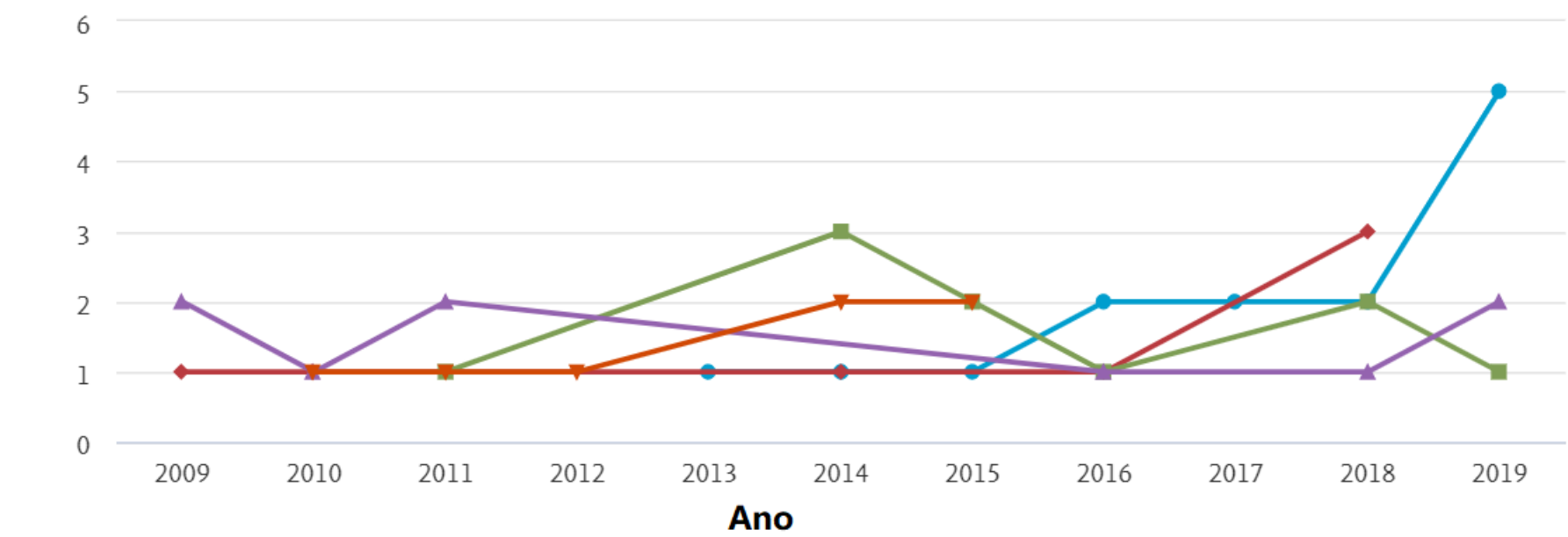

International Journal Of Supply Chain Management

International Journal Of Logistics Research And Applications

Production Planning And Control $\quad$ International Journal Of Production Economics

$*$ International Journal Of Production Research

Fonte: Elaboração própria (2019)

Durante a pesquisa na base SCOPUS, os autores se depararam com diferentes temas relacionados ao modelo SCOR. A Tabela 2 apresenta aqueles temas mais relevantes relacionados à pesquisa, enquanto a Figura 6 mostra um aspecto geral de todos os assuntos. O tema Decision Sciences é o quarto tema com maior número de publicações associadas com 98 artigos, evidenciando a relevância entre a associação de métodos de Apoio Multicritério à Decisão (AMD) e a Gestão da Cadeia de Suprimentos.

Tabela 2 - Temas relacionados ao modelo SCOR

\begin{tabular}{|l|c|c|}
\hline Tema & Quantidade & Percentual \\
\hline Business, Management \& Accouting & $\mathbf{1 5 8}$ & $\mathbf{2 2 , 3 \%}$ \\
\hline Engineering & $\mathbf{1 4 1}$ & $\mathbf{1 9 , 9 \%}$ \\
\hline Computer Science & $\mathbf{1 1 2}$ & $\mathbf{1 5 , 8 \%}$ \\
\hline Decision Sciences & $\mathbf{9 8}$ & $\mathbf{1 3 , 8 \%}$ \\
\hline Social Sciences & $\mathbf{4 1}$ & $\mathbf{5 , 8 \%}$ \\
\hline Environmental Science & $\mathbf{3 5}$ & $\mathbf{4 , 9 \%}$ \\
\hline Mathematics & $\mathbf{2 5}$ & $\mathbf{3 , 5 \%}$ \\
\hline
\end{tabular}

Fonte: Elaboração própria (2019) 
Figura 6 - Distribuição dos artigos por tema

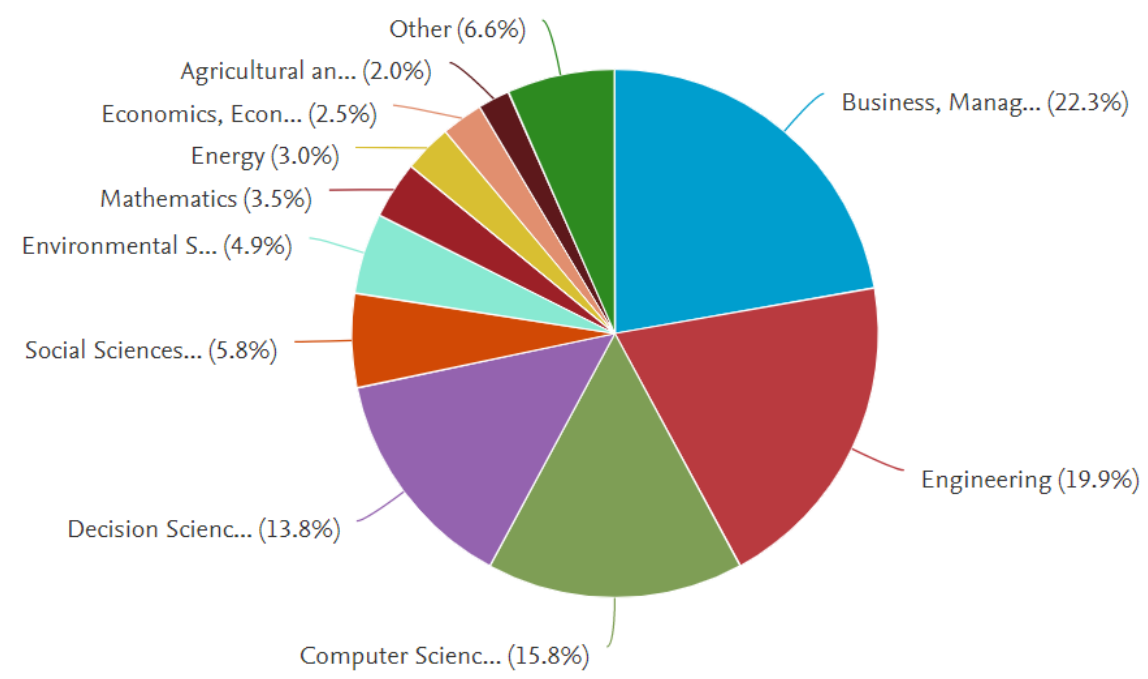

Fonte: Elaboração própria (2019)

\subsection{MÉTODO ORDINAL SAPEVO-M E PLATAFORMA SAPEVOWEB}

Com o objetivo de solucionar problemas reais, a Pesquisa Operacional adota modelos lógico-matemáticos, caracterizando-se pela sua multidisciplinariedade (SANTOS et al, 2015). A abordagem multicritério de apoio à decisão pode ser caracterizada como um conjunto de métodos que mostra um problema com mais clareza e as alternativas são avaliadas por múltiplos critérios, nos quais, em sua maioria dos casos, são conflitantes. Os métodos multicritérios de apoio à decisão são técnicas de assessoramento ao agente decisor para a tomada de decisões sobre problemas complexos, avaliando, ordenando ou selecionando alternativas mediante diferentes pontos de vista e de critérios pré-definidos (GOMES; GOMES, 2019).

O método SAPEVO-M (Simple Aggregation of Preferences Expressed by Ordinal Vectors -Multi Decision Makers) representa uma nova versão do método ordinal original SAPEVO (Gomes et al. 1997) que possibilitava a avaliação de apenas um decisor. Esta versão evoluída estende o método a múltiplos decisores, além de introduzir um processo de normalização das matrizes de avaliação, incrementando a consistência do modelo (TEIXEIRA et al., 2019).

Para os mesmos autores, o método SAPEVO-M consiste, basicamente, em dois processos. Primeiramente, a transformação ordinal da preferência entre critérios, expressada por um vetor representando os pesos dos critérios. O segundo processo é a transformação ordinal da preferência entre alternativas dentro de um determinado conjunto de critérios. As informações de preferência no SAPEVO-M são denotadas por uma série de comparações pareadas entre as alternativas. A relação entre as alternativas é expressa em uma escala de sete pontos, na qual são mensuradas, relativamente, a importância entre as alternativas. A partir da avaliação entre alternativas, é obtida uma matriz com a representação numérica correspondente. O resultado da preferência entre as alternativas é expresso pelo vetor resultante da multiplicação matricial entre o vetor peso dos critérios $\mathrm{V}$ e a matriz de avaliação das alternativas $M$. As alternativas são, então, ordenadas em ordem decrescente dos valores numéricos obtidos, gerando-se o ranking desejado.

A plataforma computacional para emprego do método SAPEVO-M denomina-se 
SapevoWeb e pode ser acessada pelo endereço: www.sapevoweb.com. Esta plataforma é um programa com uma interface gráfica amigável e apresenta resultados em tempo computacional reduzido (TEIXEIRA et al., 2018). O sistema SapevoWeb foi desenvolvido pelo Laboratório de Estudos de Governança, Gestão e Otimização (LEGGO), a partir de uma parceria entre o corpo técnico do Centro de Análise de Sistemas Navais (CASNAV), um grupo de pesquisa do Programa de Pós-Graduação em Engenharia de Produção da Universidade Federal Fluminense (UFF) e um grupo de pesquisa do Programa de PósGraduação em Engenharia de Sistemas e Computação do Instituto Militar de Engenharia (IME).

A plataforma SapevoWeb ressalta uma novidade introduzida pelo método SAPEVO-M ao expressar valores numéricos associados à ordenação por ocasião da apresentação do resultado final. Isto é, apesar do SAPEVO-M se tratar de um método ordinal multicritério e multidecisor de apoio à decisão, a informação transmitida ao decisor não se restringe à disposição das alternativas em uma ordem. Adicionalmente, o software SapevoWeb permite conhecer o quanto uma alternativa ficou melhor ordenada, relativamente, do que outra (TEIXEIRA et al., 2019).

\section{MÉTODO DE PESQUISA}

A presente pesquisa iniciou-se pela revisão de literatura de Cadeia de Suprimentos e do modelo SCOR, por meio da leitura e análise de artigos publicados nesta área. Os autores utilizaram a base de dados Scopus, acessada por meio do portal de periódicos da CAPES na internet, para acessar os artigos mais recentes e os mais citados para elaborar o referencial teórico necessário para a preparação deste artigo.

Primeiramente, em 15 de agosto de 2019, as buscas foram realizadas a partir do sítio da internet da base Scopus, utilizando-se das palavras chaves "Supply Chain" e "Supply Chain Operations Reference" e selecionando-se a opção "All Fields" no campo de busca desejado. Além disso, a busca foi limitada, por meio da ferramenta "Limit" daquela base de dados, aos artigos publicados no período entre os anos de 2009 e 2019. Nesta primeira etapa foram obtidos 304 artigos, que foram apresentados de acordo com o seu número de citações pela seleção da opção "Sort on: Cited By (highest)". Na etapa posterior, os artigos foram apresentados por data de lançamento, a partir do mais recente, por meio da opção "Sort on: Cited By (highest)". Os autores decidiram, alinhados ao objetivo do trabalho, a realizar um levantamento estatístico sobre os 304 artigos encontrados, que é apresentado na seção 2 . A leitura dos artigos para o alicerce teórico contou com uma filtragem pela leitura prévia do título e, em um segundo momento, do resumo dos mesmos.

Como já explicitado na seção anterior, o tema Decision Science se destaca quando associada à Gestão da Cadeia de Suprimentos, sendo o quarto tema com maior número de publicações nos últimos 10 anos relacionado à GCS. Entretanto, os autores decidiram verificar a correlação entre o modelo SCOR e os métodos de apoio à decisão multicritério para problemas tipicamente ordinais, cujo resultado pretendido seja o estabelecimento de um ranking. Uma pesquisa na base de dados Scopus, realizada no período de 21 a 23 de agosto de 2019 com combinações duas a duas, entre as expressões "supply chain operations reference" e as expressões "multicriteria ranking" ou "ordinal multicriteria" ou "ordinal ranking" obteve apenas um artigo como resultado. Contudo, o referido artigo não aplica nenhum método de apoio à decisão. Parente et al. (2008) apresentam um modelo interdisciplinar para pesquisa da GCS, cujo estudo foca no marketing na CS. No mesmo período, de 21 a 23 de agosto de 2019, foram realizadas buscas na base de dados Scopus, com as palavras chave "SAPEVO" ou "SAPEVO-M", mas não foi encontrado documento 
algum. Portanto, comprova-se o relativo ineditismo do trabalho em avaliar os fornecedores em uma cadeia de suprimentos por meio do método ordinal SAPEVO-M.

No presente trabalho, a empresa foco é o COMRJ e os autores utilizaram dados públicos do Portal da Transparência para selecionar as 8 empresas com maior relevância no fornecimento de material de saúde para aquela unidade no ano de 2018. Para estabelecer a importância dos fornecedores para a presente pesquisa, foi considerado o valor monetário total fornecido naquele ano. A Figura 7 representa um recorte da CS de material hospitalar para facilitar o entendimento do seu funcionamento e o escopo do estudo. No lado esquerdo da figura observam-se os principais fornecedores do COMRJ e no lado direito os seus principais clientes.

Figura 7 - Recorte da CS do COMRJ

BAXTER HOSPITALAR LTDA

costa camargo COM. DE

PRODUTOS HOSPITALARES LTDA

CRISTALIA PRODUTOS QUIMICOS

FARMACEUTICOS LTDA

ESPECIFARMA COM DE

MEDICAMENTOS E PRO

HOSPITALARES LTDA

METHABIO FARMACEUTICA DO

BRASIL LTDA

PRO-SAUDE DISTRIBUIDORA DE

MEDICAMENTOS EIREL - ME

PROVIDER PRODUTOS HOSPITALARES

E SANEANTES LTDA - EPP

SOLUMED DISTRIBUIDORA DE

MEDICAMENTOS E PRODUTOS PARA

SAUDE LTDA - EPP

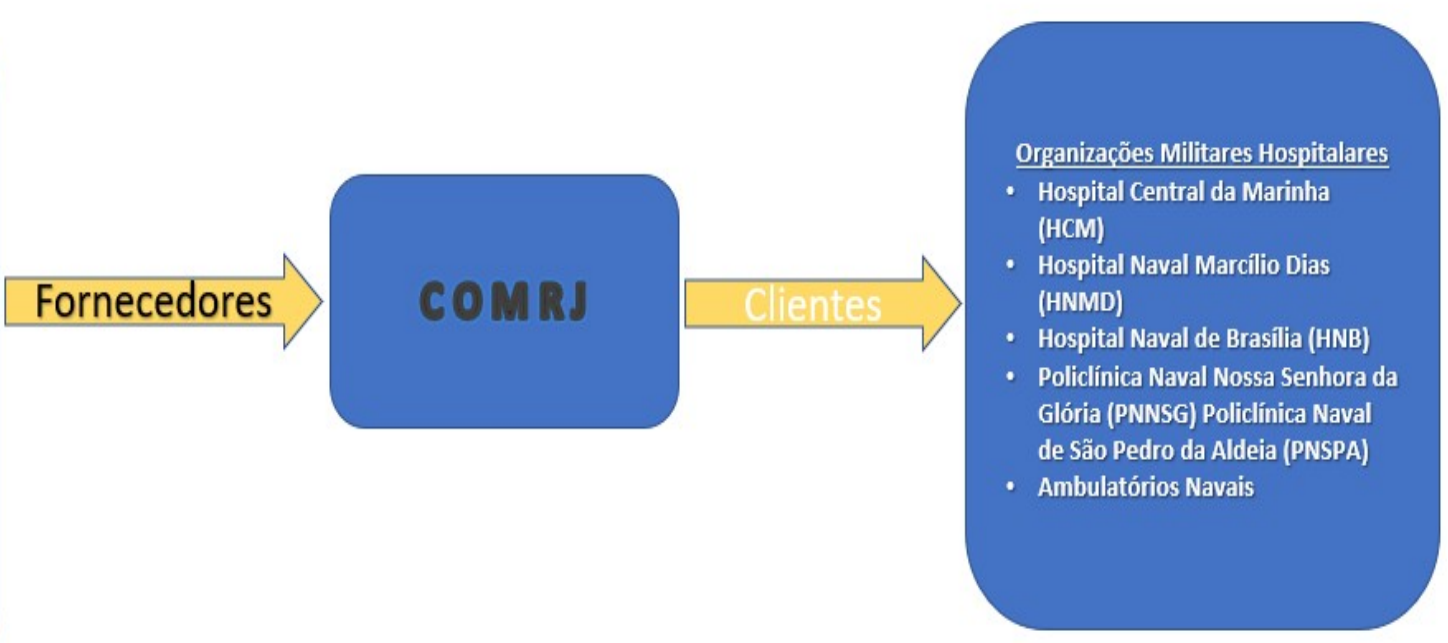

Fonte: Elaboração própria (2019)

Além disso, para melhor compreensão das particularidades desta GCS específica foram entrevistados um médico militar (Entrevistado 1 - E1) e um ex-empresário que já atuou como fornecedor de material hospitalar para a MB (Entrevistado 2 - E2). E2 é empresário aposentado com experiência de mercado no fornecimento deste tipo de material por 35 anos, os quais mais de dez anos trabalhando como fornecedor para a MB. E1 é médico anestesista, pós-graduado em Gestão em Saúde pelo COPPEAD/UFRJ, chefe de clínica do Hospital Naval de Brasília e oficial médico da MB há 20 anos. As entrevistas foram realizadas por telefone e em formato de conversa livre, em que os entrevistados eram questionados sobre a sua percepção em relação à visão acadêmica sobre GCS encontrada na literatura.

O roteiro de perguntas foi composto por perguntas abertas e fechadas. As perguntas abertas levantaram, conforme acima, o entendimento da GCS e as fechadas os critérios trazidos da publicação da APICS SCC (2017), ou seja, os atributos do modelo SCOR 12.0, que são: Agilidade (Critério 1 - C1), Responsividade (C2), Confiabilidade (C3), Gestão Eficiente (C4) e Custo (C5). Estes critérios foram avaliados em grau de importância por uma escala de sete níveis utilizada no método SAPEVO-M: absolutamente pior, muito pior, pior, equivalente, melhor, muito melhor e absolutamente melhor. Os critérios foram avaliados em comparações pareadas, assim como os fornecedores entre si, à luz de cada critério conforme 
a escala supracitada. A análise dos dados foi realizada pelo ranking e uma reflexão dessa ordenação e o quanto representa a avaliação e posicionamento dos fornecedores. Foi realizada, também, uma contraposição entre revisão de literatura e perguntas abertas.

No presente trabalho, foram consultados, ainda, dois militares (Decisor 1 - D1 - e Decisor 2 - D2) que trabalham na administração do COMRJ para a realização da avaliação dos principais fornecedores de material de saúde daquela organização militar (OM) no ano de 2018. Esta avaliação não representa a opinião oficial da MB, servindo apenas para fins acadêmicos para exemplificação do estudo de caso. Como critérios foram utilizados os atributos do modelo SCOR12.0. Os Decisores 1 e 2 foram apresentados às definições destes atributos presentes no modelo. A avaliação foi realizada diretamente na plataforma SapevoWeb, no sítio www.sapevoweb.com, por se tratar de um software de apoio à toma de decisão com base no método SAPEVO-M. D1 e D2 foram estimulados a responder o quão importante consideravam cada critério em relação aos demais, por meio de comparações dois a dois; em seguida, analogamente, avaliaram a qualidade de cada alternativa dentro de cada critério em comparação às demais.

\section{ESTUDO DE CASO: FORNECIMENTO DE MATERIAL HOSPITALAR PARA A MARINHA DO BRASIL}

O principal risco que as instituições possuem ao gerenciar o estoque numa cadeia de suprimentos é o desequilíbrio entre a demanda e a oferta. Em uma cadeia de suprimentos hospitalar, por sua vez, tal risco poderia ser mitigado pela afiliação a arranjos de sistemas "multi-hospitalares", principalmente locais. O potencial de melhoria com esta integração pode levar à economia de até $20 \%$ do custo de estoque deste sistema integrado de saúde (ZEPEDA; NYAGA; YOUNG, 2016). Dentro deste contexto, a MB se esforça para concentrar e integrar suas atividades logísticas de material hospitalar, atendendo diferentes unidades de saúde pelo seu sistema de abastecimento próprio.

Segundo as Normas para Execução do Abastecimento da Marinha do Brasil (2009), o COMRJ é um dos Órgãos de Compra do Sistema de Abastecimento da Marinha e compete a ele a execução da pesquisa e a seleção no mercado, nacional ou estrangeiro, de fornecedores. Além disso, é sua função também a aquisição dos itens para atendimento das necessidades das Organizações Militares Hospitalares, primordialmente, aquelas localizadas na área de jurisdição do Comando do $1^{\circ}$ Distrito Naval, sediado na cidade do Rio de Janeiro.

As cadeias de suprimentos hospitalares se diferem em vários aspectos das cadeias de suprimentos industriais típicas e é provável que as práticas de gerenciamento de setores como comércio e manufaturas estejam uma década à frente daquelas na área de saúde. Com a melhoria das organizações de saúde, incremento da eficiência operacional e a redução de custos, a cadeia de suprimentos hospitalar passa a concentrar mais atenção para o seu desempenho (CHENA; PRESTONA; XIAB, 2013).

Em 2018, de acordo com o Portal da Transparência, o montante de material de saúde adquirido pelo COMRJ para ser repassado às organizações militares hospitalares ou com facilidades médicas que atende, foi de, aproximadamente, R\$ 16 milhões (BRASIL, 2019). Além disso, os hospitais gerenciam grandes quantidades e variedade de itens e o armazenamento e a distribuição de todo este material são fundamentais para o fornecimento de serviços de alta qualidade ao paciente (MOONS; WAEYENBERGH; PINTELON, 2019).

Aronsson et al. (2011) questionam o porquê do atraso nas práticas de gerenciamento da cadeia de suprimentos de material de saúde, considerando que 30 a $40 \%$ das despesas hospitalares são investidas em atividades relacionadas à logística. Segundo o E2, um entrave para os hospitais públicos no Brasil seria o excesso de burocracia. Como em qualquer outro 
órgão de administração pública, todo o material deve ser adquirido mediante licitações, as quais geralmente são demasiadamente lentas.

Volland et al. (2017) atestam que a cadeia de suprimentos hospitalar muitas das vezes seria prejudicada, pois os profissionais de saúde são desviados do atendimento para exercer atividades logísticas as quais não foram preparados. O E1 refuta este posicionamento, entendendo que este não seria um problema para a Marinha, que se preocupa com a capacitação em gestão de seus médicos, à medida que vão galgando postos superiores na carreira. O mesmo entrevistado ressalta que se busca a excelência na prestação de serviços com foco na gestão, cada vez mais, visando a eficiência e eficácia nos processos. Portanto, para ele, é importante ter gestores na área da saúde que conheçam bem a atividade, sendo cada vez mais comum que médicos assistencialistas se direcionem também para funções administrativas. Assim, para o E1, estes profissionais conseguiriam ter uma visão global do assunto e tomar melhores decisões para a instituição, para os profissionais e para os pacientes.

No presente estudo de caso, os decisores D1 e D2, que trabalham na administração do COMRJ foram questionados sobre a sua percepção da importância relativa dos critérios, retirados dos atributos do modelo SCOR 12.0. Em seguida, os mesmos decisores foram demandados a avaliar o desempenho dos 8 fornecedores selecionados dentro de cada critério. As Figuras 8 e 9 ilustram a utilização do sistema online SapevoWeb para, respectivamente, a realização da avaliação dos critérios e das alternativas.

Figura 8- Estrato da página de avaliação de critérios

\section{SapevoWeb}

\section{Projeto Material Hospitalar}

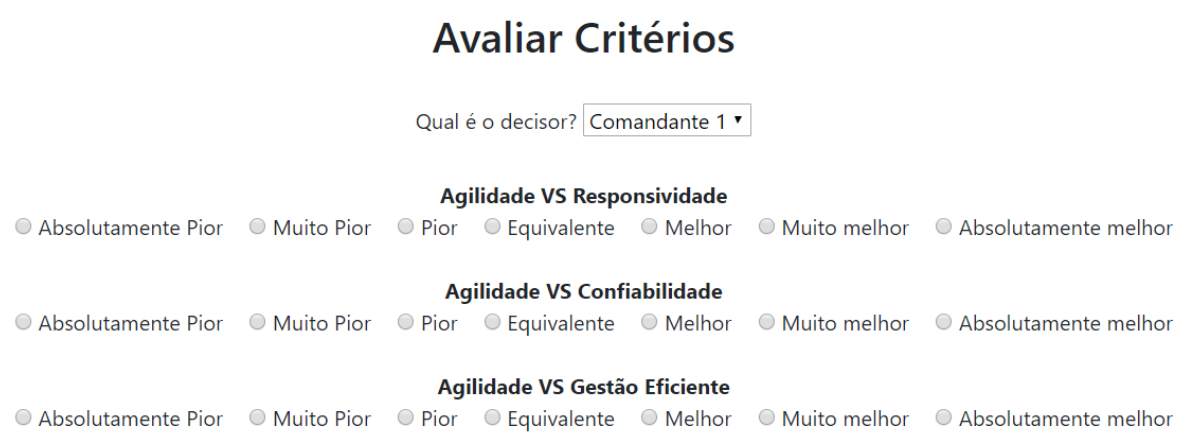

Fonte: SapevoWeb 
Figura 9 - Estrato da página de avaliação de alternativas dentro de cada critério

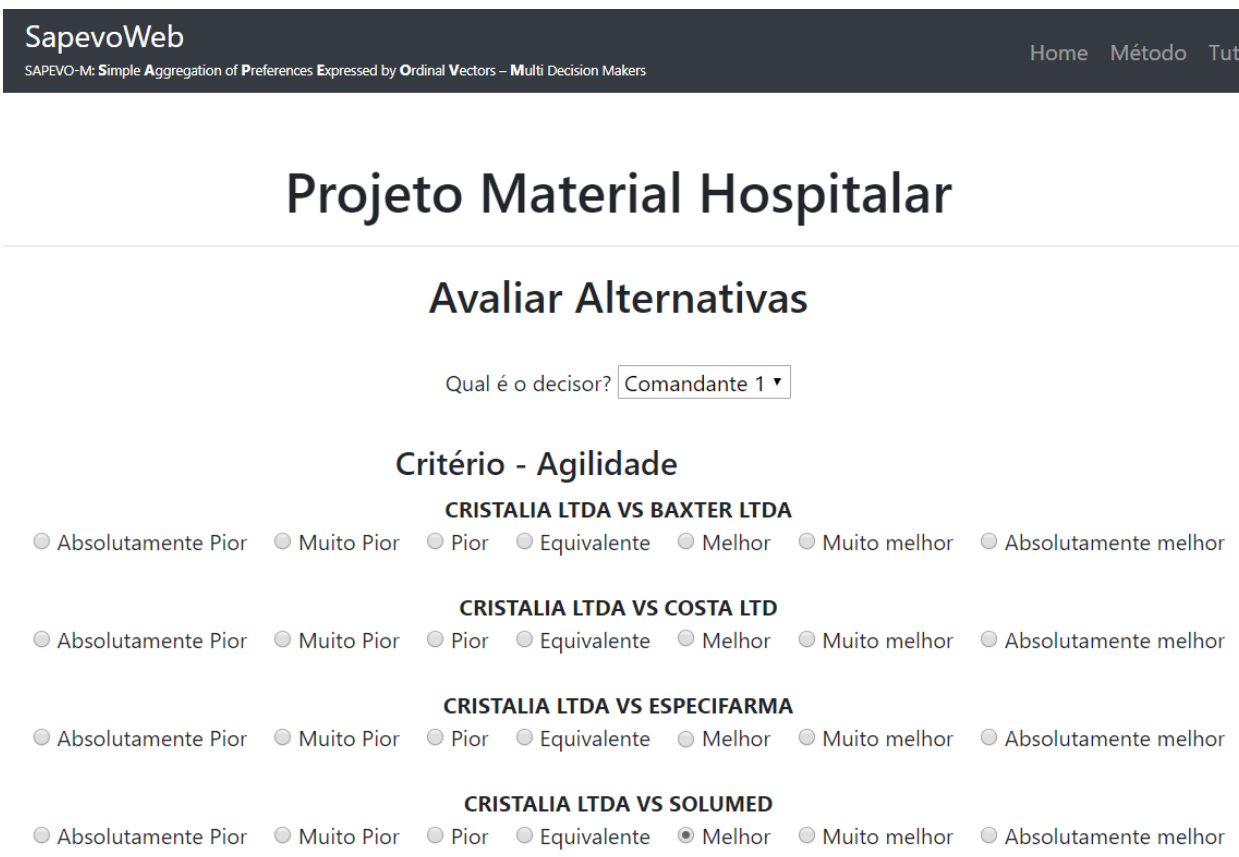

Fonte: SapevoWeb

O próprio sistema SapevoWeb calcula e apresenta os resultados: peso dos critérios, que representa a importância relativa dos mesmos para os avaliadores; e o grau obtido pelas alternativas, em ordem decrescente de pontuação. A Tabela 3 indica o valor dos pesos dos critérios calculados pelo sistema, enquanto o resultado final da ordenação é apresentado na tabela 4.

Tabela 3 - Peso dos Critérios

\begin{tabular}{|l|l|l|}
\hline Critério & Atributo & Peso \\
\hline C1 & Agilidade & $\mathbf{1 , 5 0 0 0}$ \\
\hline C2 & Responsividade & $\mathbf{0 , 7 9 2 3}$ \\
\hline C3 & Confiabilidade & $\mathbf{1 , 9 2 3 1}$ \\
\hline C4 & Gestão Eficiente de Ativos & $\mathbf{0 , 0 0 5 6}$ \\
\hline C5 & Custo & $\mathbf{0 , 8 6 1 5}$ \\
\hline
\end{tabular}

A Tabela 3 demonstra que os administradores do COMRJ consultados privilegiam, principalmente, o critério Confiabilidade da CS de material hospitalar. Provavelmente, por se tratar de material de saúde, para aqueles decisores é importante a certeza do cumprimento dos acordos celebrados para que os atendimentos não sejam prejudicados.

Tabela 4 - Ordenação das Alternativas - Fornecedores

\begin{tabular}{|l|l|l|}
\hline Ranking & Fornecedor & Grau Obtido \\
\hline $\mathbf{1}^{\mathbf{0}}$ & CRISTALIA LTDA. & $\mathbf{6 , 5 8 2 5 3}$ \\
\hline $\mathbf{2}^{\mathbf{0}}$ & BAXTER LTDA. & $\mathbf{5 , 4 6 5 7 6}$ \\
\hline $\mathbf{3}^{\mathbf{0}}$ & COSTA LTDA. & $\mathbf{5 , 3 6 3 6 7}$ \\
\hline $\mathbf{4}^{\mathbf{0}}$ & ESPECIFARMA & $\mathbf{4 , 9 6 1 0 7}$ \\
\hline $\mathbf{5}^{\mathbf{0}}$ & SOLUMED & $\mathbf{4 , 6 4 7 4 4}$ \\
\hline $\mathbf{6}^{\mathbf{0}}$ & PRO-SAUDE & $\mathbf{3 , 9 1 0 1 6}$ \\
\hline $\mathbf{7}^{\mathbf{0}}$ & PROVIDER & $\mathbf{3 , 3 8 8 1 2}$ \\
\hline
\end{tabular}


A Tabela 4 demonstra que não houve grande discrepâncias entre os graus obtidos por uma determinada empresa e àquela subsequentemente ranqueada. No caso estudado, as notas próximas podem significar não haver diferenças significativas entre o serviço prestado por uma empresa e o daquela que se segue no ranking de fornecedores de 2018. Por outro lado, como o resultado é apresentado por uma escala absoluta, pode-se observar que o $1^{\circ}$ colocado teve um desempenho próximo ao dobro do $7^{\circ}$ colocado ou que o $8^{\circ}$ colocado teve um desempenho equivalente à metade do $2^{\circ}$ colocado naquele ano. Isso significa que, se não há grandes diferenças entre uma alternativa e a seguinte, esta diferença se evidencia à medida que as mesmas se distanciam no ranking. Esta informação pode ser fundamental para um tomador de decisão, por exemplo, entender os ganhos e prejuízos na seleção de uma determinada alternativa em detrimento de outra. Em uma situação hipotética em que um fornecedor melhor colocado no ranking não possa ser selecionado por algum motivo alheio à vontade do decisor, este poderia ter maior segurança para analisar a seleção de outras alternativas do ranking.

\section{CONCLUSÃO}

O sistema SapevoWeb surge como uma ferramenta importante para que resultados de problemas complexos possam ser facilmente obtidos e os pesquisadores possam focar em outras etapas do processo. A disponibilização gratuita online desta plataforma de auxílio à decisão multicritério possibilita a difusão de um novo método ordinal para a comunidade acadêmica.

O presente estudo se propôs a utilizar um método AMD ordinal sob uma ótica diferente em um estudo de GCS, introduzindo uma nova possibilidade de abordagem para valorar aspectos da GCS por meio de ordenação. De acordo com os especialistas consultados, a empresa CRISTALIA LTDA foi a melhor colocada em um ranking de oito fornecedores de material hospitalar do COMRJ. Entretanto, ao apresentar um grau para cada alternativa ordenada, o método SAPEVO-M permite análises subjetivas sobre a diferença na qualidade do serviço de cada uma das empresas. Consequentemente, em uma situação hipotética em que aquela empresa não pudesse concorrer para ser selecionada para fornecimento de um determinado item, os clientes poderiam aceitar o $2^{\circ}$ colocado no ranking como uma alternativa viável sem prejuízo significativo na qualidade do serviço. Por outro lado, caso a opção fosse pelo último colocado do ranking, os clientes poderiam observar uma queda na qualidade do serviço apresentado, considerando que esta alternativa teve uma nota 2,5 vezes menor que a empresa que ficou em $1^{\circ}$ lugar na avaliação.

Para melhoria da gestão de fornecimento de materiais hospitalares na MB, sugere-se que esta pesquisa seja replicada com um número ainda maior de fornecedores e empregando mais especialistas do COMRJ como avaliadores e estendendo a outros setores do SSM. Pesquisas futuras podem realizar a revisão da literatura em outras bases de dados, utilizar as métricas do modelo SCOR como critérios para avaliar os fornecedores ou estender a aplicação do método SAPEVO-M a outros níveis da cadeia de suprimentos. 


\section{REFERÊNCIAS BIBLIOGRÁFICAS}

[1] APICS SCC. About APICS SCC. Disponível em: http://www.apics.org/about/overview. Acesso em: 29 de novembro de 2018.

[2] ARONSSON, Håkan; ABRAHAMSSON, Abrahamsson; SPENS, Karen. Developing lean and agile health care supply chains. Supply Chain Management: An International Journal, v. 16, n. 3, p.176-183, 2011. DOI 1108/13598541111127164

[3] BRASIL. Controladoria Geral da União. Portal da Transparência. Disponível em: http://www.portaltransparencia.gov.br/. Acesso em: 12/07/2019.

[4] BRASIL. Ministério da Defesa. Marinha do Brasil. Anuário Estatístico da Marinha do Brasil /ANEMAR 2018. Rio de Janeiro / RJ: Secretaria-Geral da Marinha. Diretoria de Administração da Marinha. v. 1, setembro, 2019.

[5] BRASIL. Ministério da Defesa. Marinha do Brasil. Carta de Serviços ao Cidadão 2019. Rio de Janeiro / RJ: Centro de Obtenção da Marinha no Rio de Janeiro. Disponível em: https://www.marinha.mil.br/comrj/sites/www.marinha.mil.br.comrj/files/ carta_COMRJ.pdf. Acesso em: 04 de setembro de 2019.

[6] BRASIL. Ministério da Defesa. Marinha do Brasil. Normas para Assistência MédicoHospitalar da Marinha do Brasil (DGPM-401). Rio de Janeiro / RJ: Diretoria Geral de Pessoal Militar, $3^{\text {a }}$ Revisão, 2012.

[7] BRASIL. Ministério da Defesa. Marinha do Brasil. Normas para Execução do Abastecimento da Marinha do Brasil (SGM - 201). Rio de Janeiro / RJ: Secretaria Geral da Marinha, 6ª Revisão, 2009.

[8] CHENA, Daniel Q.; PRESTONA, David S.; XIAB, Weidong. Enhancing hospital supply chain performance: A relational view and empirical test. Journal of Operations Management, v.31, p. 391-408, 2013.

[9] COOPER, Martha C.; LAMBERT, Douglas M.; PAGH, Janus D. Supply Chain Management: More Than a New Name for Logistics. The International Journal of Logistics Management, v. 8,n. 1, p.1-14, 1997. DOI 1108/09574099710805556

[10] COUNCIL OF SUPPLY CHAIN MANAGEMENT PROFESSIONALS. Disponível em: https://cscmp.org/. Acesso em: 24 de novembro de 2018.

[11] GOMES, Carlos Francisco Simões; RIBEIRO, Priscilla Cristina Cabral. Gestão da Cadeia de Suprimentos integrada à Tecnologia da Informação, $2^{\mathrm{a}}$ ed. Rio de Janeiro: Editora SENAC, 2014.

[12] GOMES, Luiz Flavio Autran Monteiro; GOMES, Carlos Francisco Simões. Princípios e métodos para a tomada de decisão: Enfoque multicritério, $6^{a}$ ed. São Paulo: Atlas, 2019.

[13] GOMES, Luiz F.A.M.; MURY, Antonio R.; GOMES, Carlos F.S. Multicriteria ranking with ordinal data. Systems Analysis Modelling Simulation, 1997.

[14] LI, Ling; SU, Qin; CHEN, Xu. Ensuring supply chain quality performance through applying the SCOR model. International Journal of Production Research, v. 49, p.33-57, 2011. DOI: $\underline{10.1080 / 00207543.2010 .508934}$ 
[15] MOONS, Karen; WAEYENBERGH, Geert; PINTELON, Liliane. Measuring the logistics performance of internal hospital supply chains -A literature study. Omega, Elsevier, v.82, p.205-217, 2019.

[16] NTABE, E.N.; LEBEL, L.; MUNSON, A.D.; SANTA-EULALIA, L.A. A systematic literature review of the supply chain operations reference (SCOR) model application with special attention to environmental issues. International Journal Production Economics, Elsevier, v.169, p.310-332, 2015.

[17] PARENTE, Diane H.; LEE, Peggy D. Lee; ISHMAN, Michael D.; ROTH, Aleda V. Marketing and supply chain management: a collaborative research agenda. Journal of Business \& Industrial Marketing, v.23, n.8, p.520-528, 2008. DOI 10.1108/08858620810913335

[18] PEÑA-OROZCO, Diego León; RIVERA, Leonardo. Sensitivity analysis of the SCOR metrics selected for the measurement of the management of a fruit-growing supply chain. Dyna, v.84, n.203, p. 306-315, 2017.

[19] SANTOS, Marcos; QUINTAL, Renato Santiago; PAIXÃO, Alexandre Camacho da; GOMES, Carlos Francisco Simões. Simulation of Operation of an Integrated Information for Emergency Pre-hospital Care in Rio de Janeiro Municipality. Procedia Computer Science, v. 55, p. 931-938, 2015.

DOI:10.1016/j.procs.2015.07.111.

[20] SELLITTO, Miguel Afonso; PEREIRA, Giancarlo Medeiros; BORCHARDT, Miriam; SILVA, Rosnaldo Inácio da; VIEGAS, Cláudia Viviane. A SCOR-based model for supply chain performance measurement: application in the footwear industry.

International Journal of Production Research, 2015. DOI: 10.1080/00207543.2015.1005251

[21] TEIXEIRA, L. F. H. S. B., SANTOS, M. e GOMES, C. F. S. SapevoWeb Software (v.1). (2018). Disponível em: http://www.sapevoweb.com. Acesso em: 30/07/ 2019.

[22] TEIXEIRA, Luiz Frederico Horácio de Souza de Barros; SANTOS, Marcos dos; GOMES, Carlos Francisco Simões. Proposta e implementação em python do método Simple Aggregation of Preferences Expressed by Ordinal Vectors - Multi Decision Makers: uma ferramenta web simples e intuitiva para Apoio à Decisão Multicritério. In: SIMPÓSIO DE PESQUISA OPERACIONAL E LOGÍSTICA DA MARINHA, 19., 2019, Rio de Janeiro, RJ. Anais [...]. Rio de Janeiro: Centro de Análises de Sistemas Navais, 2019.

[23] VELYCHKO, Oleksandr. Integration of SCOR-Modeling and Logistical Concept of Management in the System of Internal Transportation of Milk Cooperative. Mediterranean Journal of Social Sciences, v.6, n.1, s.2, p.14-24, 2015. DOI 10.5901/mjss.2015.v6n1s2p14

[24] VOLLAND, Jonas; FUGENER, Andreas; SCHOENFELDER, Jan; BRUNNER, Jens O. Material logistics in hospitals: A literature review. Omega, Elsevier, v.69, p.82101, 2017.

[25] ZEPEDA, E. David; NYAGA, Gilbert N.;YOUNG, Gary J. Supply chain risk management and hospital inventory: Effects of system affiliation. Journal of Operations Management, v.44, p.30-47, 2016. 